\title{
AN ENERGY-BASED ENVELOPE FUNCTION FOR THE STOCHASTIC SIMULATION OF EARTHQUAKE ACCELEROGRAMS
}

\author{
P.J.Stafford ${ }^{\star}$, S.Sgobba and G.C.Marano
}

\begin{abstract}
An energy-based envelope function is developed for use in the stochastic simulation of earthquake ground motion. The envelope function is directly related to the Arias intensity of the ground motion as well to the manner in which this Arias intensity is built-up over time. It is shown that this build-up, represented by a Husid plot, can be very well modelled using a simple lognormal distribution. The proposed envelope makes use of parameters that are commonly available in seismic design situations, either following a deterministic scenario-type analysis or following a more comprehensive probabilistic seismic hazard analysis, either in terms of Arias intensity or the more common spectral acceleration. The shape parameters of the envelope function are estimated following the calculation of the analytic envelopes for a large number of records from PEER Next Generation of Attenuation (NGA) database. The envelope may also be used to predict the distribution of peak ground acceleration values corresponding to an earthquake scenario. The distribution thus obtained is remarkably consistent with those of the recent NGA models.
\end{abstract}

\section{Introduction}

Structural and geotechnical analyses are increasingly being based upon nonlinear time-history procedures. Historically, this approach has not been widely adopted primarily for two reasons: (1) nonlinear time-history analyses are far more computationally demanding than equivalent static alternatives, and (2) there are numerous design scenarios for which it is difficult to obtain natural earthquake accelerograms that adhere to criteria such as spectrum compatibility. The first of these is

\footnotetext{
* Department of Civil \& Environmental Engineering, Imperial College London, London SW7 2AZ, UK

- Department of Environmental Engineering and Sustainable Development, Technical University of Bari, viale del Turismo 10 - 74100 Taranto (Italy) phone: +39 0994733312
} 
becoming less and less tenable as a reason for not performing nonlinear time-history analyses due to the rapid and continued increase in computational power that is readily and cheaply available. The second reason continues to exist currently but will inexorably cease to do so in the future. Although the second reason makes explicit mention of natural accelerograms, structural analyses may, at least in principle, be conducted using modified natural records (modified through adjustments in either the time or frequency domains) or with entirely simulated records. The caveat 'at least in principle' must be added here as in some countries code prescriptions will preclude the use of entirely simulated accelerograms (Beyer and Bommer, 2007). The exclusive preference for natural accelerograms is on one hand intuitive and understandable but is on the other illogically restrictive. The fact that a recorded accelerogram exists provides arguments both for and against the use of natural records. In the affirmative case, a recorded accelerogram (provided it is processed correctly) is undeniably a realistic representation of the ground shaking that occurred for a particular seismological scenario. In the negative case, the recording represents what has happened, not what will happen, and so long as uncertainty exists regarding the exact future loading it is not possible to be sure that past real accelerograms will more accurately reflect future loading than simulated accelerograms. In reality, the arguments against the use of simulated accelerograms are not offered for philosophical reasons but rather because it is justifiably thought that current approaches to simulating accelerograms do not result in sufficiently realistic ground motions. Here, when talking about simulated accelerograms we refer to stochastic-based methods rather than detailed seismological models that are very rarely used as inputs for structural analyses. The primary purpose of this article is to advance this situation by offering a new envelope function that represents the first component of a full nonstationary stochastic approach to accelerogram generation.

Rezaeian and Der Kiureghian (2008) have recently discussed the fact that there are essentially two types of stochastic ground-motion model; referred to as 'source-based' and 'site-based' models. Source-based models are conceptually attractive as they allow physical parameters obtained from seismological studies to be directly incorporated into the simulation process (Zerva, 1988; Quek et al., 1990). Although the ability to incorporate physical constraints from such seismological studies is appealing, stochastic models for generating accelerograms are most commonly required for regions of low-to-moderate seismicity and in such regions these studies may not have been conducted. This is a significant disadvantage of the source-based approach as these physical parameters have been shown to vary rather significantly from region to region (Chouet et al., 1978). Site-based models, on the other hand, do not require detailed seismological information and are 
therefore more readily applicable to regions where very few instrumental recordings (either seismograms or accelerograms) have been made. One disadvantage of the latter approach, however, is that the specific characteristics of a particular seismological scenario can not always be accounted for, e.g., the commonly adopted power spectral density function proposed by Kanai (1957) and Tajimi (1960) has a shape that is only dependent upon properties of the site but not of the source.

The approach taken in the present study is to define a new envelope function that is directly compatible with an overall seismic design process. Apart from certain academic exercises, timehistory analyses are conducted for the purposes of identifying actions that must be accommodated during structural or geotechnical design. Seismic design codes throughout the world allow varying levels of rigour to be exercised when determining appropriate design actions. At the simplest level, design actions are prescribed as equivalent static loads that may be read directly from a code spectrum (normally some form of, or approximation to, a uniform hazard spectrum, UHS). Beyond this level, time-history analyses may be conducted using accelerograms compatible with the spectrum just mentioned or a site-specific hazard analysis may be conducted that will result in an alternative design spectrum to which accelerograms may be scaled or matched. If one follows the first option for time-history analysis of using the code spectrum then the information that one will typically have are spectral amplitudes corresponding to a particular return period. In most cases, hazard disaggregation results are not available and information related to which seismological scenarios are dominating the hazard will not be available. As Naeim and Lew (1995) point out, for locations where different seismological scenarios contribute significantly to the UHS at different response periods, scaling or matching to a code spectrum inevitably leads to accelerograms that are either very rare or not physically reasonable. That said, for most projects, even if one is working with a code spectrum, it should be possible to independently identify the key seismic sources that could affect the site and thus obtain information such as likely magnitude and distance values that may then be considered as design scenarios. Of course, if a site-specific analysis is conducted then such scenarios should be identified routinely during the analysis. Therefore, for deterministic scenario-based analyses, or for analyses following on from a probabilistic seismic hazard analysis (either from a code or a site-specific case), one should always be able to infer a magnitude, a source-to-site distance, and some characteristics regarding the site conditions in addition to the corresponding amplitudes of response spectral ordinates. The assumption that such information is available is pivotal to the methodology that is presented herein. 
It is well known that earthquake ground-motion is a classic example of a nonstationary stochastic process with temporal variation of both its amplitude and frequency characteristics. However, it is also generally acknowledged that for many applications the component of the process that dominates amplitude-based measures of structural response, such as drift, may be represented by a weakly stationary process (Soong and Mircea, 1993). The most common approach to simulating earthquake accelerograms therefore consists of starting with a Gaussian stationary white noise process $w(t)$ which passes through a linear filter in order to obtain the desired power spectral density function and the result is then multiplied by a deterministic envelope function $e(t)$ so that the stationary filtered white noise $a_{s t}$ gets the desired time-dependent amplitude (Figure 1).

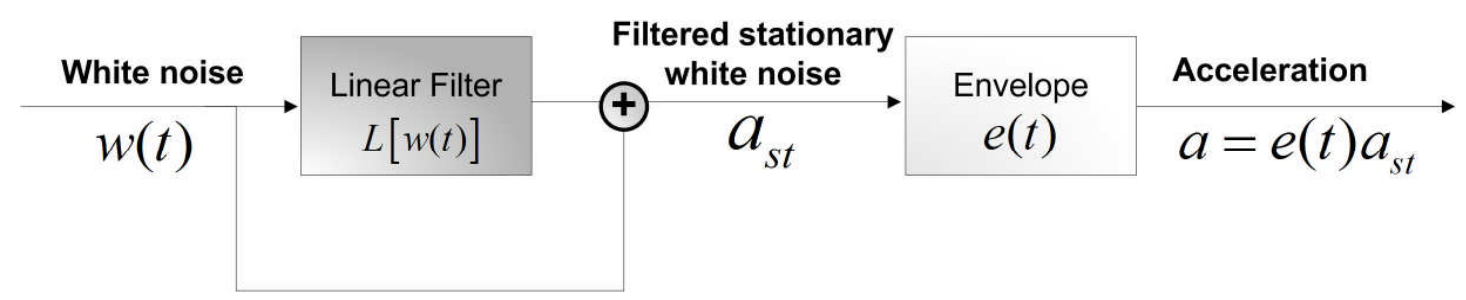

Figure 1: Typical processs for stochastic ground-motion simulation

In Figure 1 the specification of the frequency content of the ground motion is made through the generic linear filter, $L[]$, but in most stochastic analyses the frequency content is prescribed directly in terms of the power spectral density function (PSDF) of the process. The simulation of accelerograms using this power spectral representation has recently been outlined rigorously by Liang et al. (2007).

Many different envelope functions have been proposed over the past 40 years or so; the most common of which are discussed by Jangid (2004). Quek et al. (1990) suggest that, so long as the envelope function is able to appropriately reflect the energy content of the ground shaking, estimates of structural response are not particularly sensitive to the actual window function used. However, these authors were only concerned with the estimation of pseudo-spectral velocities and for other measures of structural response these findings may not hold. Even if measures of the structural response are relatively insensitive to the windowing function it is still preferable to make this component of the stochastic model as realistic as possible. This way when the response due to real and simulated accelerograms coincide we can be confident that we are modelling the ground motions appropriately rather than achieving our end through inappropriate means. Therefore, the objective herein is to develop an envelope function that is as realistic as possible while also 
ensuring that the energetic characteristics of the accelerogram are incorporated in a quantitative manner.

In the present study, the envelope function that is developed is related directly to the seismological and ground-motion parameters that are routinely specified during a design situation. The envelope function is tied directly to the expected Arias intensity of the accelerogram given some seismological scenario as well as to two shape parameters that are in turn related to seismological parameters. The information that is required in order to derive the envelope therefore consists of information regarding the scenario, i.e., magnitude, distance, average shear-wave velocity, etc., as well as an estimate of the Arias intensity associated with this particular combination of seismological parameters. This information may be specified directly during a scenario-based analysis, where one simply defines the seismological parameters from the outset, or via a probabilistic seismic hazard analysis (PSHA), where the most relevant seismological parameters are identified following disaggregation of the ground-motion level corresponding to a particular return period (Bazzurro and Cornell, 1999). Figure 2 provides a schematic overview of the three main avenues via which one may obtain the information required to implement the envelope function that is presented herein. Note that, as mentioned previously, where the analyst desires an envelope function that is consistent with some code spectrum then the option of a PSHA conducted for a spectral acceleration encapsulates this situation as even though the code will not directly provide information regarding hazard-consistent earthquake scenarios, it is very unlikely that suitable scenarios will not be able to be inferred indirectly.

It should be noted that, in Figure 2, two options related to PSHA are presented. The first case corresponds to a situation in which the PSHA is conducted directly in terms of Arias intensity whereas the second case corresponds to the most common case where the PSHA is conducted in terms of spectral acceleration. For the purpose of deriving the envelope function of this study, the first option is the most direct. However, it is currently rather rare for PSHA to be conducted in terms of Arias intensity despite the existence of some empirical predictive models that have been derived for this purpose (e.g., Travasarou et al., 2003; Stafford et al., 2008). This is not restrictive at all however, as the second approach, based upon PSHA conducted for spectral acceleration, actually leads to a more accurate prediction of the envelope function provided that the correlation between spectral acceleration and Arias intensity is accounted for. More detail will be provided regarding all three of the possible options in Figure 2 in what follows. 


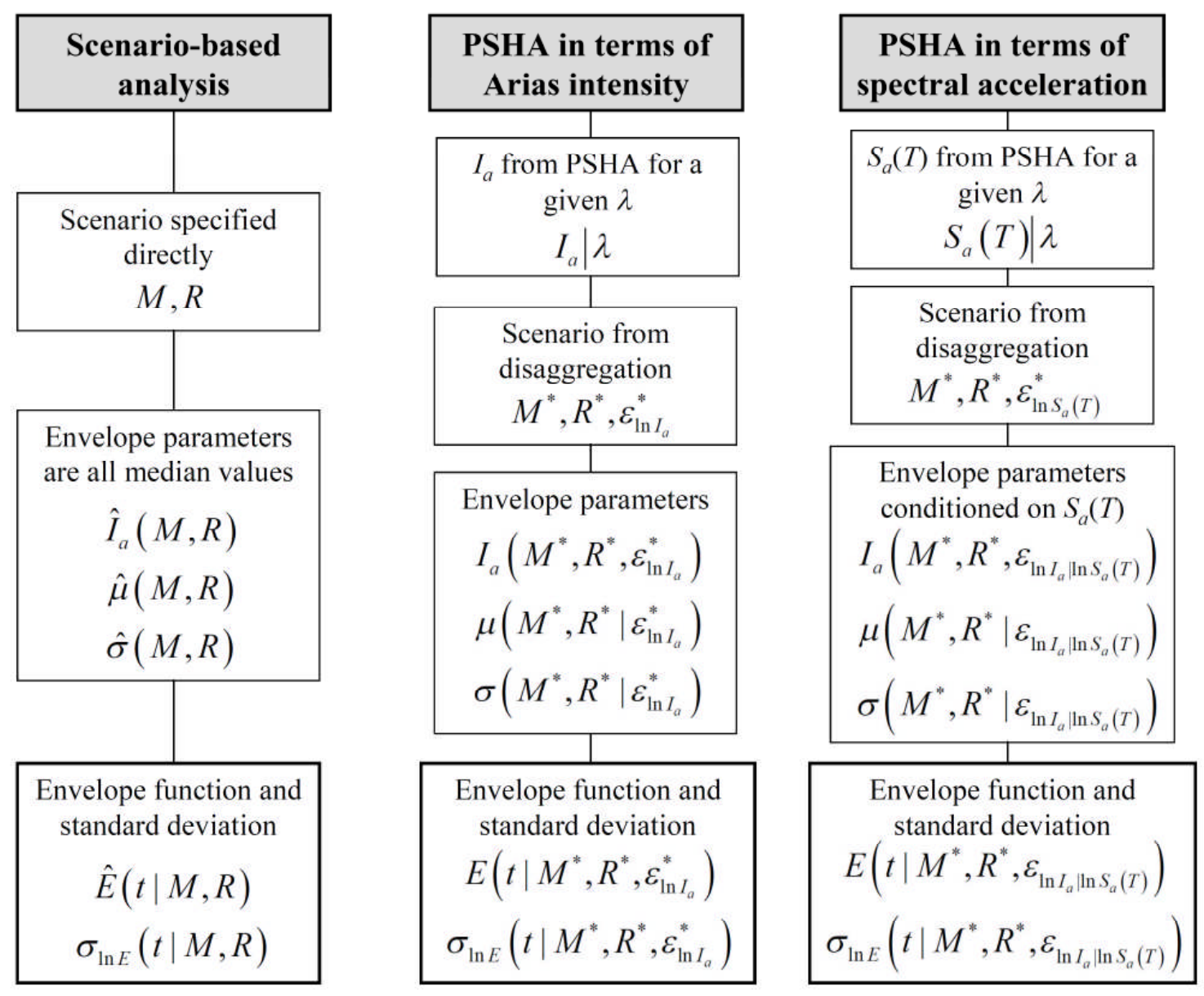

Figure 2: Schematic overview of the process via which one obtains an envelope function, $E(t \mid \ldots)$, and associated (logarithmic) standard deviation, $\sigma_{\ln E}(t \mid \ldots)$, for particular design situations. For the cases involving PSHA, $\lambda$, is some predefined annual rate of exceedance of the ground motion $I_{a}$ or $S_{a}(T)$ as the case may be. The parameters $\mu$ and $\sigma$ are generic envelope shape parameters that will be defined in what follows.

\section{Theoretical formulation of the Husid Envelope Function (HEF)}

The form of the envelope function is arrived at through consideration of the manner in which energy is temporally distributed throughout an accelerogram. A key objective of this study is to ensure that the envelope that is presented is consistent with energy characteristics of real accelerograms. Here, this consistency is monitored through the use of Arias intensity, both in terms of the total Arias intensity of a record as well as the manner in which this total Arias intensity is achieved. The Arias intensity describes the cumulative energy per unit weight absorbed by an infinite set of single-degree-of-freedom oscillators having fundamental frequencies uniformly 
distributed in $(0, \infty)$ (Travasarou et al., 2003). The Arias intensity is proportional to the integral of the squared acceleration time-history and is most commonly defined as:

$$
I_{a}=\frac{\pi}{2 g} \int_{0}^{\infty} a^{2}(t) d t
$$

where $a(t)$ is the acceleration time-history (see Stafford et al. 2008 for a more thorough definition). The temporal accumulation of Arias intensity is best viewed using a Husid plot (either in an absolute or normalized form). In this study it is convenient to make use of the normalized Husid function which may be defined as in Equation (2).

$$
H(t)=\frac{\int_{0}^{t} a^{2}(t) d t}{\int_{0}^{\infty} a^{2}(t) d t}=\frac{\pi}{2 g I_{a}} \int_{0}^{t} a^{2}(t) d t
$$

The form of the envelope function comes from the realisation that the function $H(t)$ satisfies all of the required conditions to allow it to be regarded as a cumulative probability distribution function and that, furthermore, the shape of $H(t)$ for real accelerograms is strikingly similar to that of the cumulative distribution function (CDF) of the lognormal distribution (see Figure 3).
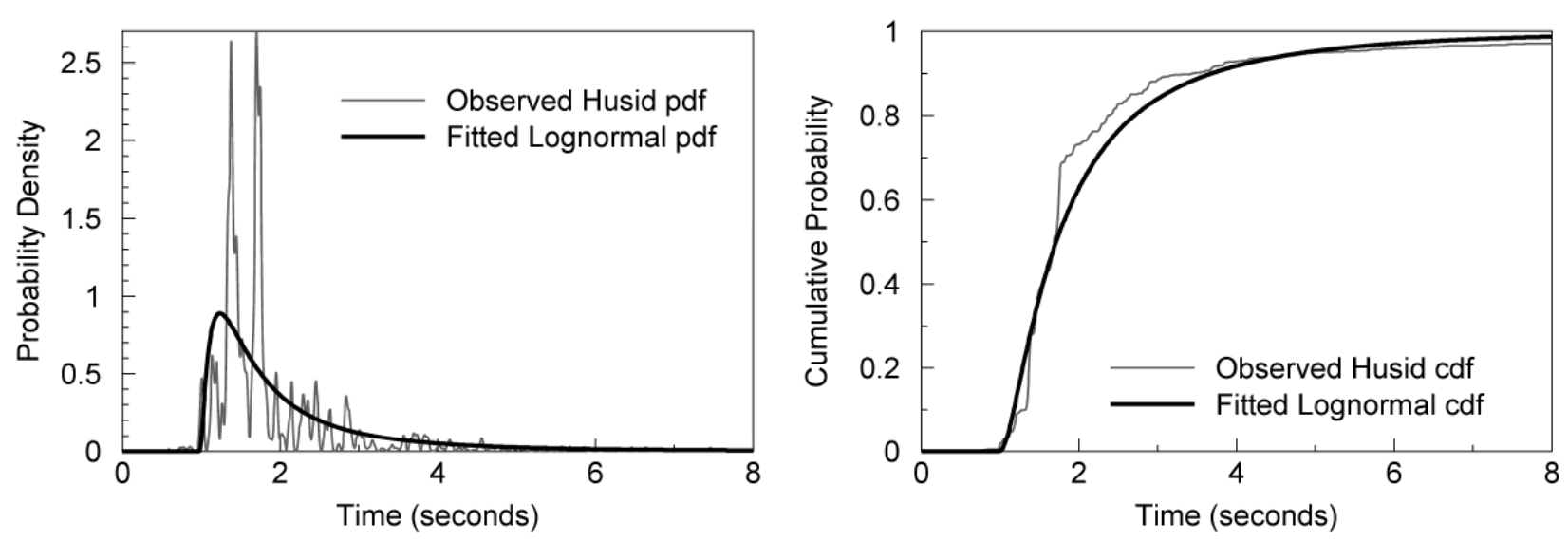

Figure 3: Example of the typical fit that may be achieved by modelling the observed husid pdf as a lognormal distribution. Note that the fitted model (both pdf and cdf correspond to the same fitted model) has been obtained from the observed Husid pdf via a maximum likelihood approach. Note also that the fitted lognormal distribution has been temporally shifted such that the origin coincides with the time at which $0.5 \%$ of the final Arias intensity for the record has been accumulated. 
This realisation leads one to suspect that a suitable candidate shape for an envelope function for accelerograms may be closely related to the probability density function equivalent of the normalized Husid function which is defined as in Equation (3).

$$
h(t)=\frac{d H(t)}{d t}=\frac{\pi}{2 g I_{a}} a^{2}(t)
$$

In order to derive the form of the envelope function we simply assume that the function $h(t)$ may be modelled using the probability density function (pdf) of the lognormal distribution. This lognormal pdf is directly related to the shape of the envelope and requires the specification of two parameters that correspond to the mean and standard deviation of the distribution. In this study we define these shape parameters as functions of common seismological parameters. Given the shape of the envelope we must then scale this generic shape to ensure that the absolute amplitude of the envelope is consistent with the amplitudes that we would expect for a particular scenario. In order to derive relationships to define the shape parameters, as well as to ensure that we achieve the correct amplitudes for the envelope, we must make direct comparisons with the envelopes of real accelerograms.

The first step in the derivation thus requires the determination of the envelopes associated with a set of real accelerograms. The accelerograms that have been used in this study consist of records from the strong-motion database of the NGA project (Chiou et al., 2008). Not all of the available records have been considered in this study with the particular subset that has been adopted being exactly the same as that which has previously been used by Stafford et al. (2008a). For specific details regarding the rationale behind selecting this particular subset of accelerograms the interested reader is referred to Stafford et al. (2008a). Here, it suffices to say that 2406 records (4812 individual horizontal components) from 114 earthquakes were included in the dataset for the present study. These records are associated with earthquakes having moment magnitudes ranging from 4.79 to 7.9 and depths to the top of their rupture ranging from zero to $14.5 \mathrm{~km}$. The recordings from these earthquakes are made at sites with average shear-wave velocities over the upper $30 \mathrm{~m}$ between 116 and $2017 \mathrm{~m} / \mathrm{s}$ and at locations ranging from 0.07 to $100 \mathrm{~km}$ from the assumed rupture surface of the events.

There exist a number of definitions for the envelope of a random process. The three most notable of which are those due to Rice (1954), Crandall and Mark (1963) and Dugundji (1958), which is often 
attributed to Cramer and Leadbetter (1967). The Rice (1954) envelope is based upon the expansion of the process $x(t)$ about some central frequency. This is often considered to be the classical definition of the envelope, and, among other things, it has been used in the past to derive the statistical properties of ocean waves and second order wave forces. The envelope of Crandall and Mark (1963) is an "energy envelope" and is defined in terms of $x(t)$ and its derivative $\dot{x}(t)$. This definition of the envelope is used in stochastic averaging techniques, which are used in conjunction with the Fokker-Planck-Kolmogorov equation to determine the statistics of non-linear random vibration. The envelope of Dugundji (1958) is derived from the random process $x(t)$ and its Hilbert transform $\hat{x}(t)$ and it is this definition that we adopt in this work. The Dugundji envelope has been used to tackle the first passage problem in random vibration, and the original definition has now been extended to include non-stationary random processes (Langley, 1986). The calculation of the envelope of an accelerogram using the approach of Dugundji (1958) consists of two steps: (1) calculating the pre-envelope of the accelerogram, and (2) calculating the envelope itself from the pre-envelope. The pre-envelope of an acceleration time-history, $a(t)$, is defined as in Equation (4).

$$
z(t)=a(t)+i \hat{a}(t)
$$

Here, the pre-envelope is a complex-valued signal that has the original acceleration time-history as its real component and the Hilbert transform of the original time-history as its imaginary component ( $i=\sqrt{-1}$ and is the complex unit). The Hilbert transform, $\hat{a}(t)$, of $a(t)$ is given by the operation in Equation (5).

$$
\hat{a}(t)=\frac{1}{\pi} \int_{-\infty}^{\infty} \frac{a(\tau)}{t-\tau} d \tau
$$

The concept of the pre-envelope has proven to be useful for determining the spectral moments of non-stationary processes by Di Paola (1985). Di Paola demonstrated that the covariance structure of the process defined by the pre-envelope can be used to establish time-domain interpretations of stationary spectral moments and that non-stationary spectral characteristics may also be defined in an analogous way. 
Given the pre-envelope of the original accelerogram one may compute the envelope by taking the modulus of the complex process. That is, the envelope may be calculated using Equation (6).

$$
E(t)=\sqrt{a^{2}(t)+\hat{a}^{2}(t)}
$$

An example of a real envelope, calculated according to the above procedure is shown in Figure 4.

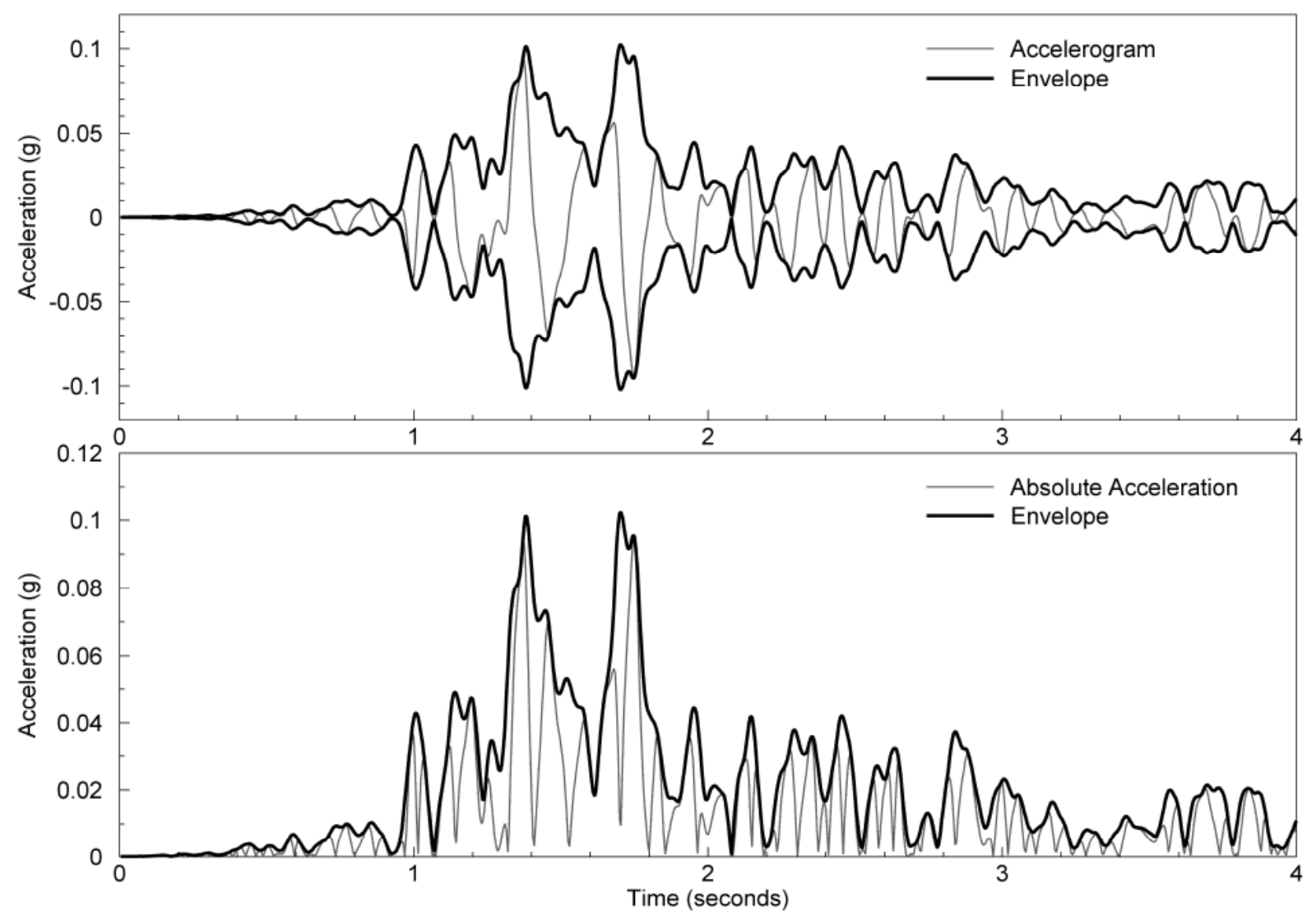

Figure 4: Longitudinal component of the Golden Gate Park recording of the $\mathrm{M}_{\mathrm{w}} 5.281957$ San Francisco earthquake and its associated envelope, as computed using the procedure outlined in the text. Note that only the first four seconds of the record are shown.

The Hilbert transform of the accelerogram, $\hat{a}(t)$, is a signal that has the same amplitude spectrum and frequency content as the original signal but with a consistent $90^{\circ}$ phase shift applied to all components (Marple, 1999). This fact proves to be particularly useful for deriving the general form of the envelope function. From Equation (6) and the knowledge that the integral of a linear combination of functions is the linear combination of the integrals of the individual functions, one may obtain the following expression: 


$$
\int_{-\infty}^{\infty} E^{2}(t) d t=\int_{-\infty}^{\infty} a^{2}(t) d t+\int_{-\infty}^{\infty} \hat{a}^{2}(t) d t
$$

Now recalling Parseval's theorem that dictates equivalence of power between the time and frequency domains as formally stipulated by Equation (8),

$$
\int_{-\infty}^{\infty}|x(t)|^{2} d t=\int_{-\infty}^{\infty}|X(f)|^{2} d f
$$

we may state that the two integrals on the right-hand-side of Equation (7) are equal (as the Hilbert transform acts only to introduce a phase shift and does not influence the amplitude spectrum) and that Equation (7) may therefore be rewritten as in Equation (9).

$$
\int_{-\infty}^{\infty} E^{2}(t) d t=2 \int_{-\infty}^{\infty} a^{2}(t) d t
$$

Now, recalling Equation (3) we may arrive at the general expression for the envelope function, $E(t)$, to be used in this study.

$$
E(t)=\sqrt{\frac{4 g I_{a}}{\pi} h(t)}
$$

The Husid pdf, given by $h(t)$, is assumed to be modelled by the pdf of a lognormal distribution which leads to the final expression for the envelope function:

$$
E(t)=\sqrt{\frac{4 g I_{a}}{t \sigma \pi \sqrt{2 \pi}} \exp \left[-\frac{(\ln (t)-\mu)^{2}}{2 \sigma^{2}}\right]}
$$

Hence, the Husid Envelope Function (HEF) is completely defined for all time provided that one has estimates of the Arias intensity corresponding to the scenario in question as well as estimates of the shape parameters $\mu$ and $\sigma$ that correspond to the mean and standard deviation of the lognormal distribution. The estimates of the Arias intensity may be made using existing empirical models such as that of Travasarou et al. (2003). The shape parameters must be related to the seismological 
characteristics of the scenario through new empirical predictive models. The derivation of such models is the subject of the following section.

\section{Empirical models for the shape parameters}

For every component in the dataset, the Arias intensity was calculated which then allowed Equation (11) to be rearranged into a function that could be directly compared with a lognormal distribution. The optimal shape parameters for every component were then obtained using standard maximum likelihood techniques as implemented in the MATLAB® function 'lognfit'. The shape parameters for every component were then associated with the metadata for each component which consisted of the most typical seismological parameters that define scenarios for seismic design; namely, the moment magnitude, the rupture distance, the average shear-wave velocity and the depth to the top of the rupture. Nonlinear random-effects regression analyses were then conducted using the 'nlme' package of R (Pinheiro et al., 2008; R Development Core Team, 2008) in order to obtain empirical models for the shape parameters.

There is no precedent for functional forms for estimating these shape parameters, although one might anticipate that the shape parameter $\mu$ would scale in a similar manner to many duration measures (Bommer et al., 2008) and that the parameter $\sigma$ would, at least, be expected to be a function of the average shear-wave velocity (Dobry et al., 1978) as well as of distance. Numerous functional forms were considered and the relative performance of each candidate was assessed in terms of standard statistical metrics such as the Akaike Information Criterion, the Bayesian Information Criterion, the likelihood ratio test, and the residual variance. The coefficients of the various candidate models were all checked to ensure that they were statistically significant at the 95\% confidence level and that strong correlations among these coefficients did not exist. The regression analysis was conducted on the shape parameters corresponding to the individual groundmotion components. The final functional forms are given in Equations (12) and (13) while the coefficients of these models are presented in Table 1.

$$
\begin{gathered}
\mu=b_{1}+b_{2} M_{w}+\left(b_{3}+b_{4} M_{w}\right) \ln \sqrt{R_{r u p}^{2}+b_{5}^{2}}+b_{6} \ln V_{S, 30}+b_{7} Z_{t o r} \\
\ln \sigma=c_{1}+c_{2} R_{r u p}+c_{3} \ln V_{S, 30}+c_{4} Z_{\text {tor }}
\end{gathered}
$$


The performance of these two models may be assessed through inspection of the residuals in Figures 5 and 6 for the shape parameters $\mu$ and $\sigma$ respectively. It may be appreciated from both Equations (12) and (13) as well as Figures 5 and 6 that the shape parameter $\mu$ is normally distributed marginally while the shape parameter $\sigma$ is lognormally distributed marginally. This information, along with the prior knowledge that the Arias intensity is also lognormally distributed enables us to derive an expression for the variance of the envelope as detailed in the next section.

Table 1: Parameter values and associated $95 \%$ confidence intervals (defined as \pm values) for the empirical models for the shape parameters of Equation (12) (left two columns) and Equation (13) (right two columns). The shape parameter $\mu$ is normally distributed marginally so the parameters relate directly to the prediction

of this shape parameter whereas the shape parameter $\sigma$ is lognormally distributed and these parameter values therefore correspond to the prediction of $\ln \sigma$ as in Equation (13) The standard deviation terms are defined as follows: $\sigma_{\mathrm{E}}$ is the inter-event standard deviation, $\sigma_{\mathrm{A}}$ is the intra-event standard deviation including the component-to-component variability, $\sigma_{\mathrm{C}}$ is the component-to-component standard deviation, $\sigma_{1}$ is the intra-event standard deviation excluding the component-to-component variability, $\sigma_{\mathrm{T}, \mathrm{ARB}}$ is the total standard deviation for an arbitrary component, and $\sigma_{\mathrm{T}, \mathrm{GM}}$ is the total standard deviation corresponding to the geometric mean of two horizontal components. See Baker and Cornell (2006a) for a more detailed discussion.

\begin{tabular}{|c|c|c|c|}
\hline \multicolumn{2}{|c|}{ Shape parameter $\mu$} & \multicolumn{2}{c|}{ Shape parameter $\sigma$} \\
\hline Parameter & Estimate & Parameter & Estimate \\
\hline $\mathrm{b}_{1}$ & $-6.0391 \pm 0.7769$ & $\mathrm{c}_{1}$ & $0.3151 \pm 0.1139$ \\
\hline $\mathrm{b}_{2}$ & $1.0895 \pm 0.1188$ & $\mathrm{c}_{2}$ & $-0.003 \pm 0.0003$ \\
\hline $\mathrm{b}_{3}$ & $1.8415 \pm 0.1316$ & $\mathrm{c}_{3}$ & $-0.0957 \pm 0.0165$ \\
\hline $\mathrm{b}_{4}$ & $-0.2065 \pm 0.0193$ & $\mathrm{c}_{4}$ & $-0.0104 \pm 0.0096$ \\
\hline $\mathrm{b}_{5}$ & $5.7575 \pm 1.356$ & & \\
\hline $\mathrm{b}_{6}$ & $-0.1383 \pm 0.0217$ & & \\
\hline $\mathrm{b}_{7}$ & $-0.0239 \pm 0.0198$ & & $0.1774 \pm 0.0286$ \\
\hline$\sigma_{\mathrm{E}, \mu}$ & $0.3269 \pm 0.0491$ & $\sigma_{\mathrm{E}, \ln \sigma}$ & $0.2244 \pm 0.0045$ \\
\hline$\sigma_{\mathrm{A}, \mu}$ & $0.2936 \pm 0.0059$ & $\sigma_{\mathrm{A}, \ln \sigma}$ & $0.0821 \pm 0.0023$ \\
\hline$\sigma_{\mathrm{C}, \mu}$ & $0.1078 \pm 0.003$ & $\sigma_{\mathrm{C}, \ln \sigma}$ & $0.2089 \pm 0.005$ \\
\hline$\sigma_{1, \mu}$ & $0.2731 \pm 0.0065$ & $\sigma_{1, \ln \sigma}$ & $0.2861 \pm 0.0181$ \\
\hline$\sigma_{\mathrm{T}, \mathrm{ARB}, \mu}$ & $0.4394 \pm 0.0367$ & $\sigma_{\mathrm{T}, \mathrm{ARB}, \ln \sigma}$ & $0.274 \pm 0.0189$ \\
\hline$\sigma_{\mathrm{T}, \mathrm{GM}, \mu}$ & $0.4259 \pm 0.0379$ & $\sigma_{\mathrm{T}, \mathrm{GM}, \ln \sigma}$ & \\
\hline & & & \\
\hline
\end{tabular}



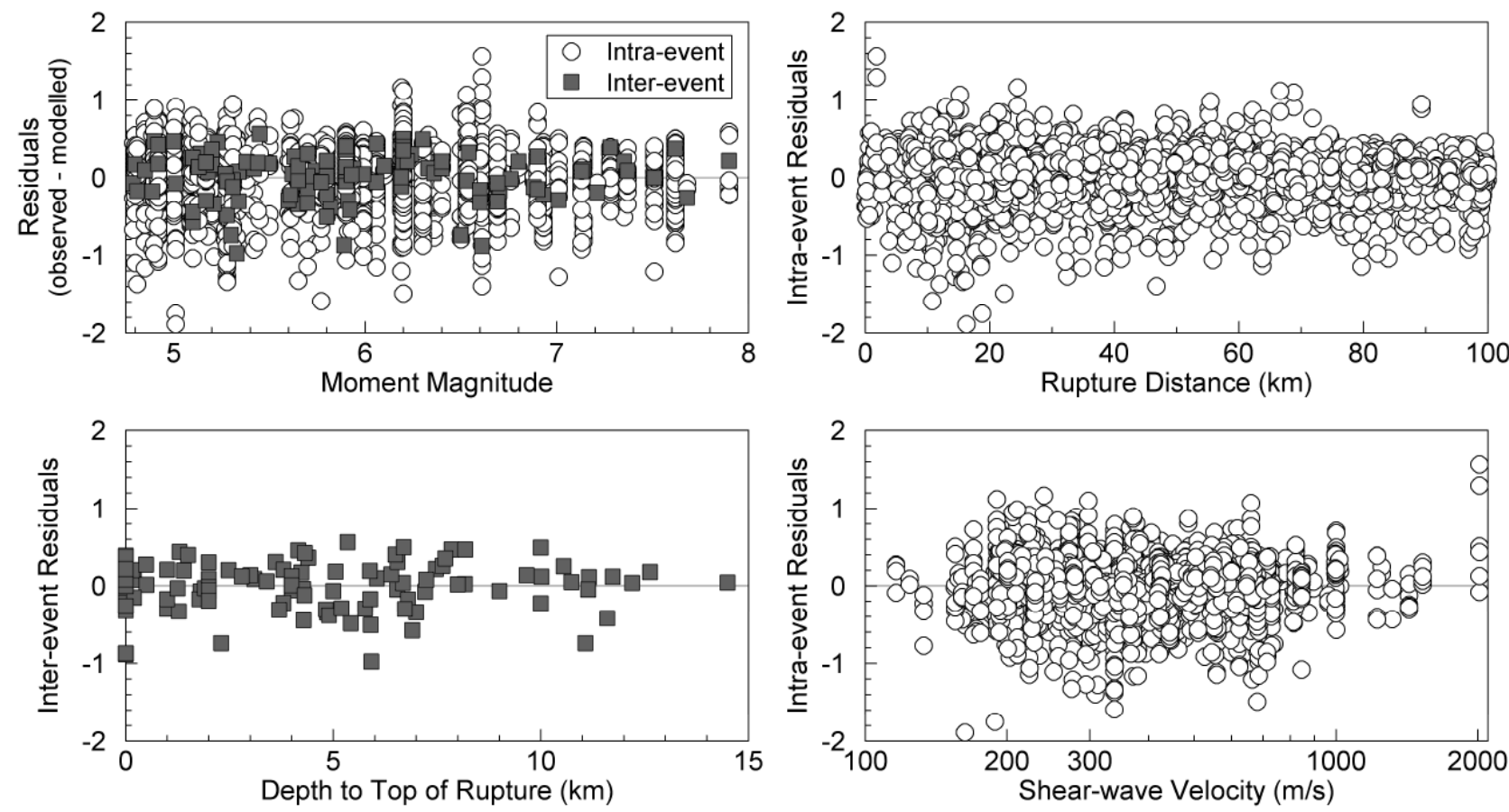

Figure 5: Residuals of the empirical model for predicting the envelope shape parameter $\mu$, i.e., residuals from the prediction of $\mu$ as in Equation (12).
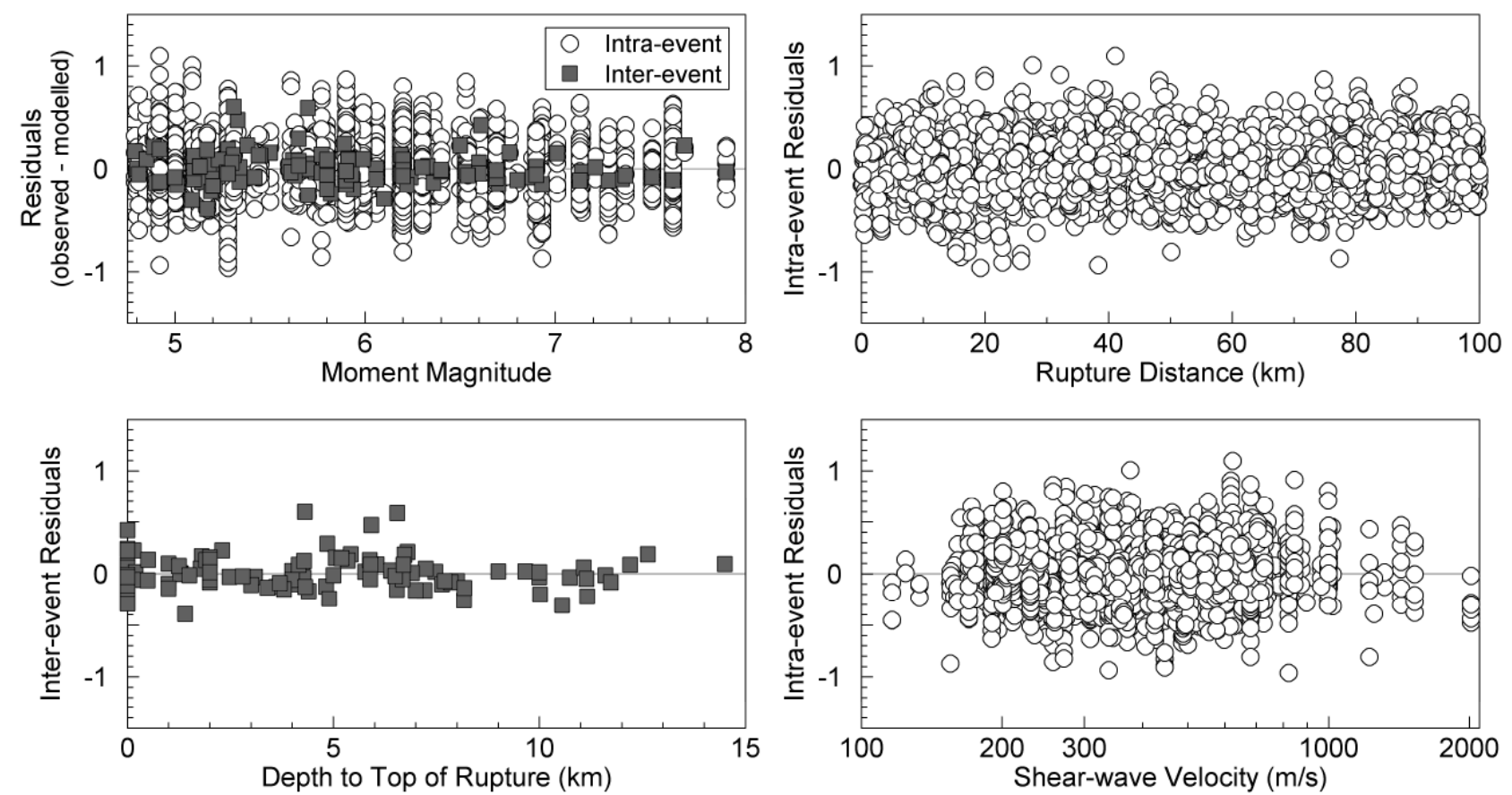

Figure 6: Residuals of the empirical model for predicting the logarithm of the envelope shape parameter $\sigma$, i.e., residuals from the prediction of $\ln \sigma$ as in Equation (13). 


\section{Envelope Variance}

In addition to the expected shape of the envelope for a given scenario it is also very desirable to obtain an estimate of the variance. As just mentioned, it is possible to derive an expression for the variance of the envelope given the knowledge that the individual parameters of $I_{a}, \mu$ and $\sigma$ are marginally lognormally, normally, and lognormally distributed respectively. Given the form of Equation (11) the central limit theorem would suggest that the envelope itself should be approximately lognormally distributed for any given time (Benjamin and Cornell, 1970). In order to test this assumption a simple Monte Carlo experiment was undertaken whereby 10,000 combinations of $I_{a}, \mu$ and $\sigma$ were drawn from their joint distribution for a given seismological scenario. The scenario that was chosen consisted of an $M_{w} 7.0$ surface-rupturing earthquake. The site of interest has an average shear-wave velocity of $760 \mathrm{~m} / \mathrm{s}$ and the distance between this site and the nearest point on the rupture surface is $20 \mathrm{~km}$. The marginal distribution of Arias intensity for this scenario was estimated using the empirical model of Travasarou et al. (2003) while the distributions of the shape parameters was made using the models presented herein. In order to ensure that any correlations among these parameters was accounted for the correlations among the total residuals for each of $\ln I_{a}, \mu$ and $\ln \sigma$ were first computed and used to construct a full covariance matrix that was then used for generating the 10,000 samples. The correlation matrix is given in Equation (14). This correlation matrix is general for all scenarios but the covariance matrix will vary with the scenario as the standard deviation of the logarithmic Arias intensity values is amplitude, magnitude and site-class dependent.

$$
\boldsymbol{\rho}=\left[\begin{array}{ccc}
\rho_{\ln I_{a}, \ln I_{a}} & \rho_{\ln I_{a}, \mu} & \rho_{\ln I_{a}, \ln \sigma} \\
\rho_{\mu, \ln I_{a}} & \rho_{\mu, \mu} & \rho_{\mu, \ln \sigma} \\
\rho_{\ln \sigma, \ln I_{a}} & \rho_{\ln \sigma, \mu} & \rho_{\ln \sigma, \ln \sigma}
\end{array}\right]=\left[\begin{array}{ccc}
1 & -0.1104 & -0.0241 \\
-0.1104 & 1 & -0.2328 \\
-0.0241 & -0.2328 & 1
\end{array}\right]
$$

From Equation (14) it may readily be appreciated that the correlations among parameters are generally very weak and may well be neglected. However, for the purposes of the current Monte Carlo exercise, retaining the correlations adds no complexity to the analysis and allows one to make a more rigorous test regarding the lognormality, or otherwise, of the distribution of envelope values for a given scenario.

In order to derive an analytical expression for the standard deviation of the envelope function we first take the logarithm of Equation (11) which leads to Equation (15). 


$$
\ln E(t)=\frac{1}{2}\left[\ln \left(\frac{4 g}{t \pi \sqrt{2 \pi}}\right)+\ln I_{a}-\ln \sigma-\frac{(\ln (t)-\mu)^{2}}{2 \sigma^{2}}\right]
$$

From this point we may see that the variance of the logarithmic envelope values may be found by propagating the variances associated with the Arias intensity and the shape parameters. Although we have recently seen that the correlations among the three parameters of $\ln I_{a}, \mu$ and $\ln \sigma$ are relatively weak, for the derivation we will act under the assumption that these cannot be neglected. The variance of the logarithmic envelope amplitudes can thus be written as:

$$
\begin{aligned}
\sigma_{\ln E(t)}^{2} \cong & \left(\frac{\partial \ln E}{\partial \ln I_{a}}\right)^{2} \sigma_{\ln I_{a}}^{2}+\left(\frac{\partial \ln E}{\partial \mu}\right)^{2} \sigma_{\mu}^{2}+\left(\frac{\partial \ln E}{\partial \ln \sigma}\right)^{2} \sigma_{\ln \sigma}^{2} \\
& +\left(\frac{\partial \ln E}{\partial \ln I_{a}}\right)\left(\frac{\partial \ln E}{\partial \mu}\right) \rho_{\ln I_{a}, \mu} \sigma_{\ln I_{a}} \sigma_{\mu}+\left(\frac{\partial \ln E}{\partial \ln I_{a}}\right)\left(\frac{\partial \ln E}{\partial \ln \sigma}\right) \rho_{\ln I_{a}, \ln \sigma} \sigma_{\ln I_{a}} \sigma_{\ln \sigma} \\
& +\left(\frac{\partial \ln E}{\partial \mu}\right)\left(\frac{\partial \ln E}{\partial \ln \sigma}\right) \rho_{\mu, \ln \sigma} \sigma_{\mu} \sigma_{\ln \sigma}
\end{aligned}
$$

While Equation (16) appears to be a rather unwieldy expression, the partial derivatives of Equation (15) are particularly simple and the expression for the variance may be obtained in a relatively compact manner. If we first make the following substitution,

$$
K_{C}=\frac{(\ln (t)-\mu)^{2}}{\sigma^{2}}
$$

one finds that Equation (16) reduces to the following:

$$
\sigma_{\ln E(t)}^{2} \cong \frac{1}{4}\left[\begin{array}{l}
\sigma_{\ln I_{a}}^{2}+K_{C} \sigma_{\mu}^{2}+\left(K_{C}-1\right)^{2} \sigma_{\ln \sigma}^{2}+\frac{\sqrt{K_{C}}}{\sigma} \rho_{\ln I_{a}, \mu} \sigma_{\ln I_{a}} \sigma_{\mu} \\
+\left(K_{C}-1\right) \rho_{\ln I_{a}, \ln \sigma} \sigma_{\ln I_{a}} \sigma_{\ln \sigma}+\left(K_{C}-1\right) \frac{\sqrt{K_{C}}}{\sigma} \rho_{\mu, \ln \sigma} \sigma_{\mu} \sigma_{\ln \sigma}
\end{array}\right]
$$

Figure 7 shows a comparison between the full analytical solution, derived above under the assumption that the amplitude of envelope values is lognormally distributed and the results of the 
10,000 Monte Carlo simulations. While there appears to be a very small bias in the estimate of the mean amplitude, as is best appreciated from inspection of the cumulative plots, the general agreement in terms of the form of the distribution is excellent. We can therefore use the analytical expression for the standard deviation of the logarithmic envelope values with a great deal of confidence. Note that, given the weakness of the correlations among the parameters, a very good approximation to the full analytical expression for the envelope variance may be given by the simple expression given by Equation (19).

$$
\sigma_{\ln E(t)}^{2} \cong \frac{1}{4}\left[\sigma_{\ln I_{a}}^{2}+K_{C} \sigma_{\mu}^{2}+\left(K_{C}-1\right)^{2} \sigma_{\ln \sigma}^{2}\right]
$$
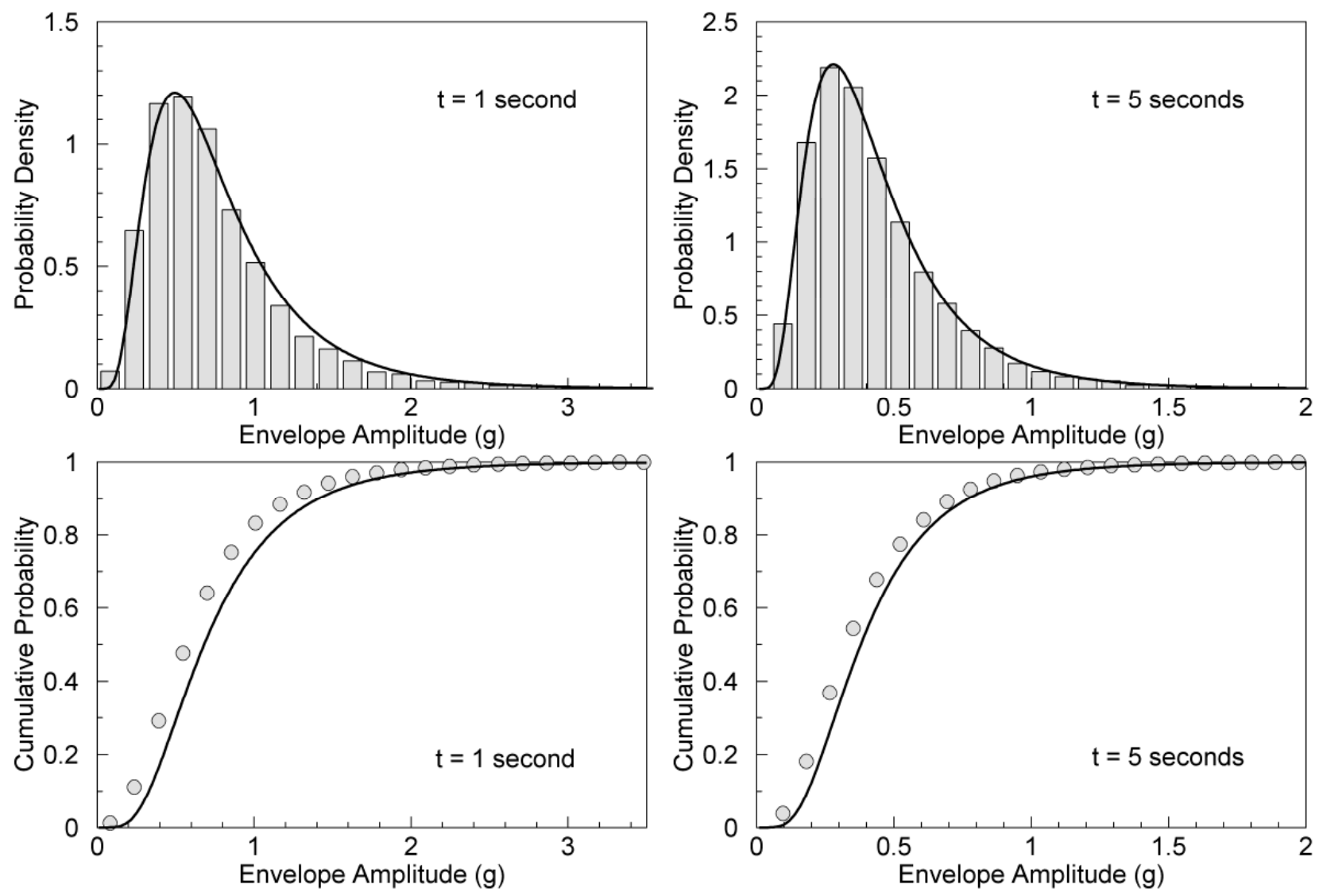

Figure 7: Comparison of the distributions of envelope amplitudes at two particular times ( 1 and 5 seconds). The bars and circles represent the results from 10,000 Monte Carlo samples drawn using the correlation matrix of Equation (14) while the solid curves represent the analytical model derived under the assumption that the envelope amplitudes are lognormally distributed. The seismological parameters for the scenario shown are: $\mathrm{M}_{\mathrm{w}} 7.0, \mathrm{Z}_{\text {tor }}=0 \mathrm{~km}, \mathrm{R}_{\text {rup }}=20 \mathrm{~km}$, and $\mathrm{V}_{\mathrm{S}, 30}=760 \mathrm{~m} / \mathrm{s}$. 


\section{Example Performance}

The preceding sections have outlined a full working model that enables one to predict the envelope of an acceleration time-series for a given seismological scenario. Figure 2 demonstrated that there were essentially three paths that one may follow to go from a design scenario through to the estimation of the envelope. Further details of how one would go about implementing each of these three alternative paths are provided here.

For the case of the deterministic scenario, the implementation of the model presented herein is straightforward, one simply takes the seismological parameters that define the scenario and insert these into an empirical model for Arias intensity, such as that of Travasarou et al. (2003), and the models presented herein for the shape parameters. From these three models one can directly obtain an estimate of the envelope function and its variance (the variance of the logarithm of the envelope) using Equations (11) and (18) (Equation (19) if the correlations are neglected).

For the second case where a PSHA has been carried out directly in terms of Arias intensity one will know the seismological scenario(s) that is dominating the hazard for a particular return period from disaggregation and one will also know the value of epsilon, $\varepsilon_{\ln I_{a}}$, consistent with this dominant scenario. Given this epsilon value the appropriate Arias intensity is completely defined, and the shape parameters that should be used within Equations (11) and (18) are given by the following, in which $\hat{I}_{a}, \hat{\mu}$, and $\hat{\sigma}$ are the median estimates of the three parameters for the scenario identified during the disaggregation.

$$
\begin{array}{cc}
\ln I_{a}=\ln \hat{I}_{a}+\varepsilon_{\ln I_{a}} \sigma_{\ln I_{a}} & \sigma_{\ln I_{a}}\left(\varepsilon_{\ln I_{a}}\right)=0 \\
\mu=\hat{\mu}+\rho_{\mu, \ln I_{a}} \varepsilon_{\ln I_{a}} \sigma_{\mu} & \sigma_{\mu}\left(\varepsilon_{\ln I_{a}}\right)=\sigma_{\mu} \sqrt{1-\rho_{\mu, \ln I_{a}}^{2}} \\
\ln \sigma=\ln \hat{\sigma}+\rho_{\ln \sigma, \ln I_{a}} \varepsilon_{\ln I_{a}} \sigma_{\ln \sigma} & \sigma_{\ln \sigma}\left(\varepsilon_{\ln I_{a}}\right)=\sigma_{\ln \sigma} \sqrt{1-\rho_{\ln \sigma, \ln I_{a}}^{2}}
\end{array}
$$

Note that because the correlations are relatively weak, the parameter values that are obtained in Equation (20) (with the exception of the Arias intensity) will be very similar to the expected values of these parameters for the given scenario. The standard deviation of the logarithmic Arias intensity for this case is zero as it is defined by the PSHA. As the variance of the envelope function is dominated by the variance of the Arias intensity the standard deviation of the envelope function is significantly reduced when a PSHA is conducted directly in terms of the Arias intensity. In this case 
we are describing the envelope function using shape parameters that are conditioned upon both the seismological parameters defined from the disaggregation as well as the actual value of Arias intensity for which the disaggregation was conducted.

For the final, and most common case, where an envelope is required that is consistent with the results of a PSHA carried out in terms of spectral acceleration (including peak ground acceleration) the dominant scenario will again be found from disaggregation. However, in this case we obtain the epsilon with respect to the spectral acceleration and must relate this to the value of the Arias intensity. Fortunately, Baker (2007) has provided the tools that are required to meet this end by formulating a model for the correlation among Arias intensity and spectral ordinates. This model is reproduced here for ease of reference as Equation (21).

$$
\rho_{\ln I_{a}, \ln S_{a}(T)}=\left\{\begin{array}{lll}
0.344-0.152 \ln (T) & \text { if } & 0.05 \leq T<0.11 \\
0.971+0.131 \ln (T) & \text { if } & 0.11 \leq T<0.40 \\
0.697-0.166 \ln (T) & \text { if } & 0.40 \leq T \leq 5.00
\end{array}\right.
$$

Note that the correlation between the Arias intensity and the peak ground acceleration is 0.82 according to Baker (2007) and that it is reasonable to interpolate between this value at that for a period of 0.05 seconds if the correlation for very short periods is required.

Now, given the above information one can formulate a set of expressions to define the parameters that should be used in Equations (11) and (18) as was previously done in Equation (20). The relevant equations for this case are given in Equation (22).

$$
\begin{array}{cc}
\ln I_{a}=\ln \hat{I}_{a}+\rho_{\ln I_{a}, \ln S_{a}(T)} \varepsilon_{\ln S_{a}(T)} \sigma_{\ln I_{a}} & \sigma_{\ln I_{a}}\left(\varepsilon_{\ln S_{a}(T)}\right)=\sigma_{\ln I_{a}} \sqrt{1-\rho_{\ln I_{a}, \ln S_{a}(T)}^{2}} \\
\mu=\hat{\mu}+\rho_{\mu, \ln I_{a}} \rho_{\ln I_{a}, \ln S_{a}(T)} \varepsilon_{\ln S_{a}(T)} \sigma_{\mu} & \sigma_{\mu}\left(\varepsilon_{\ln S_{a}(T)}\right)=\sigma_{\mu} \sqrt{1-\rho_{\mu, \ln I_{a}}^{2} \rho_{\ln I_{a}, \ln S_{a}(T)}^{2}} \\
\ln \sigma=\ln \hat{\sigma}+\rho_{\ln \sigma, \ln I_{a}} \rho_{\ln I_{a}, \ln S_{a}(T)} \varepsilon_{\ln S_{a}(T)} \sigma_{\ln \sigma} & \sigma_{\ln \sigma}\left(\varepsilon_{\ln S_{a}(T)}\right)=\sigma_{\ln \sigma} \sqrt{1-\rho_{\ln \sigma, \ln I_{a}}^{2} \rho_{\ln I_{a}, \ln S_{a}(T)}^{2}}
\end{array}
$$

As in Equation (20), the effect of knowing $\varepsilon_{\ln S_{a}(T)}$ is very weak in terms of its ability to change the values the shape parameters and their standard deviations. However, a very significant reduction in the standard deviation of the logarithmic Arias intensity can be made if one possesses knowledge of the $\varepsilon_{\ln S_{a}(T)}$ value as the correlation between Arias intensity and spectral acceleration is very strong. 
As mentioned previously, the expressions for the variance of the logarithmic envelope amplitudes given in Equations (18) and (19) are very heavily dominated by the standard deviation of the logarithmic Arias intensity. Therefore, in the case where the seismological scenario is defined from a disaggregation of a PSHA conducted for spectral acceleration the significantly reduced value of $\sigma_{\ln I_{a}}\left(\varepsilon_{\ln S_{a}(T)}\right)$ leads to a very robust estimate of the envelope function.

To demonstrate the relative performance of the predictive model for the envelope function when information is or is not known about the level of spectral acceleration corresponding to the scenario two example predictions are provided in Figure 8. For the purpose of demonstrating the impact of the knowledge of the spectral acceleration the examples have simply been taken as the first two longitudinal components in the dataset that was considered for the derivation of the envelope function, i.e., the two longitudinal components with the lowest record identification numbers in the NGA dataset. In both cases the plots on the right-hand-side of Figure 8 correspond to a case where the Arias intensity and the shape parameters are conditioned upon knowledge of the value of $\varepsilon_{\ln S_{a}(T)}$ for $\mathrm{T}=1 \mathrm{~s}$. In order to calculate the epsilon values for $\ln S_{a}(T=1 s)$ the true acceleration response spectra of the records were calculated and the epsilon value was predicted using the empirical ground-motion model of Abrahamson and Silva (2008) and the appropriate metadata for each record. The relevant metadata is presented in Figure 8. It is immediately obvious that the knowledge of the epsilon value for spectral acceleration enables one to make a far more informed prediction of the envelope function for a given scenario as well as allowing the standard deviation of this estimate to be significantly reduced.

The example fits that are presented in Figure 8 are fairly typical of the quality of fit that may be obtained through the use of the model presented herein. However, in numerous cases the implementation of the model will lead to envelopes that do not resemble the actual accelerograms. This is to be expected given just how variable the shapes of accelerograms are in reality. In the present study, this variability has been recognised from the outset so the emphasis has been upon trying to derive a relatively simple functional form and to then quantify the uncertainty as well as possible. One feature of the fits that are shown in Figure 8 is that the envelopes are not able to encompass the initial wave arrivals. As is mentioned in the caption of Figure 8, for the purpose of these comparative plots, the envelope functions have been temporally shifted such that their origins coincide with the time at which $0.5 \%$ of the total Arias intensity of the real record has been 
accumulated. This practice is followed purely because each record in the considered dataset has a differing amount and amplitude of noise prior to the actual ground shaking and some objective temporal origin must therefore be defined for both estimating the model parameters and making comparisons such as those shown in Figure 8. The initial wave arrivals that are not encapsulated by the envelope therefore represent a very minor contribution to the energy, and hence engineering significance, of the ground shaking. When it comes to the practical implementation of the envelope function presented herein, this issue associated with the temporal shift of the envelope is of no importance whatsoever.
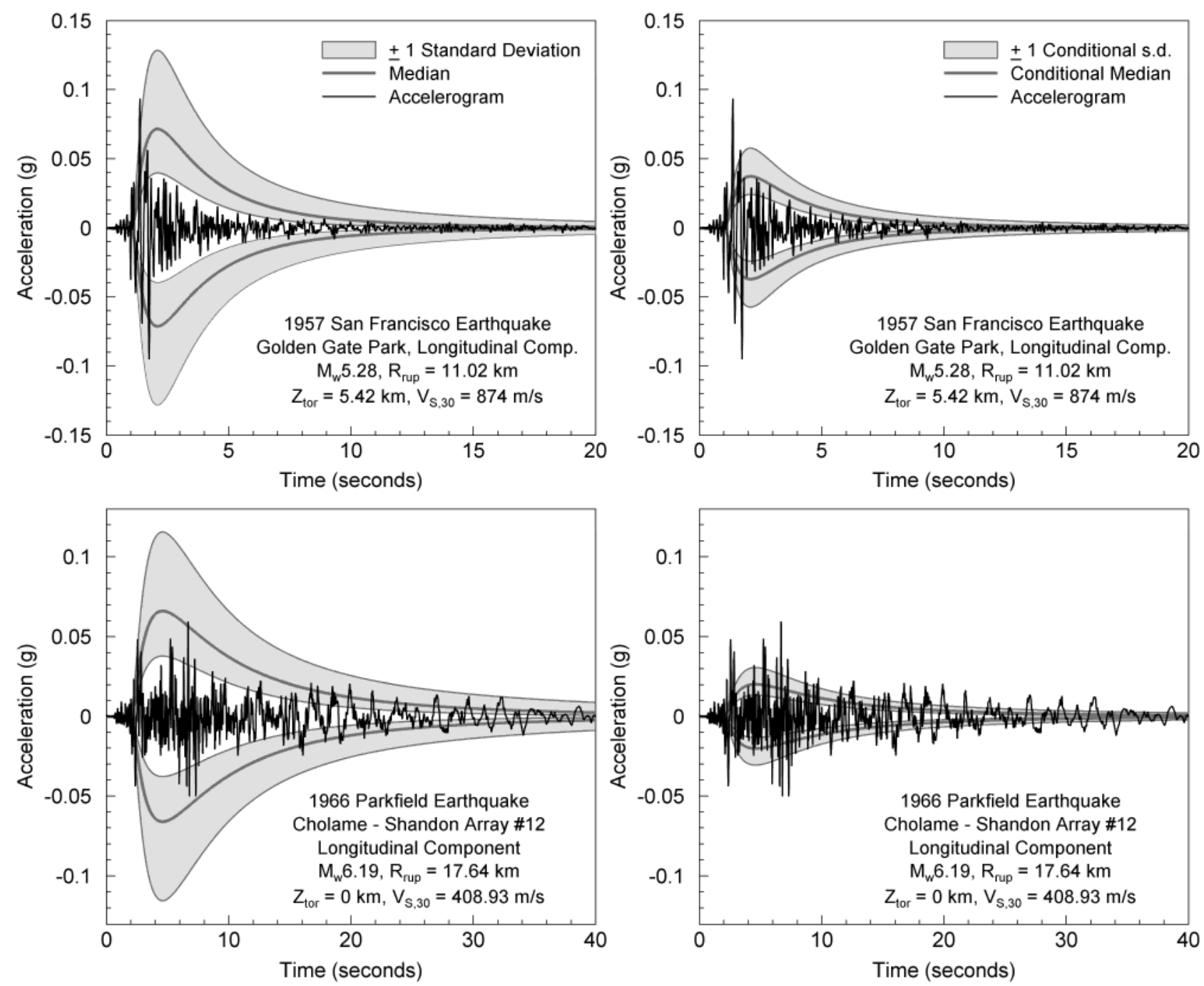

Figure 8: Example predictions of the envelope function for two components of ground motion. The top two panels relate to a recording of the 1957 San Francisco earthquake while the bottom two panels relate to a recording of the 1966 Parkfield event. The two panels on the left-hand-side correspond to the scenario-based analysis case of Figure 2, while the two panels on the right-hand-side correspond to the PSHA in terms of spectral acceleration case. In all cases, the shaded region indicates the amplitude range corresponding to plus

or minus one standard deviation from the median prediction. For the panels on the right-hand-side, the median and standard deviation are both conditional values. In both cases the fitted envelope functions have been temporally shifted such that the origin coincides with the time at which $0.5 \%$ of the final Arias intensity for each record has been accumulated. 
One may appreciate from inspection of Figure 8 that although the envelope appears to do a good job of modelling the general shape of the accelerogram, it is not able to encapsulate the peak ground acceleration. Often, these peak acceleration values correspond to rather transient spikes and will not have a significant impact upon structural or geotechnical response. However, there are still numerous methods that make use of estimates of the peak ground acceleration and for this reason it is desirable to provide a mechanism via which peak acceleration values may be related to the envelope function. If one computes the ratio of the peak acceleration and the maximum of the envelope function for all of the components in the dataset one finds that these ratios are wellmodelled by a lognormal distribution as shown in Figure 9.

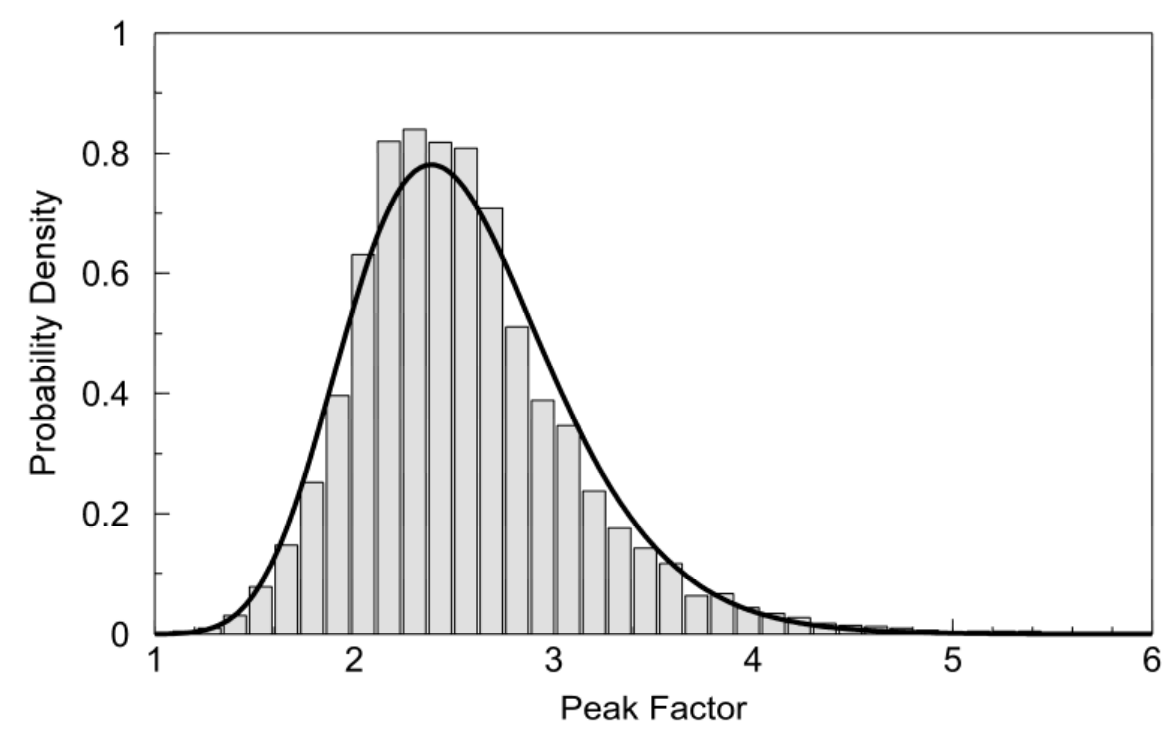

Figure 9: Distribution of the peak factor and a fitted lognormal distribution.

In Figure 9 a lognormal distribution has been plotted along with the actual peak values observed during the derivation of the envelope function. The mean value of the peak factor is very close to 2.5 (the actual value is 2.4934) and the logarithmic standard deviation is 0.2095 . No significant correlation was found between the values of the peak factor and the various seismological parameters that are used to derive the envelope function. Therefore, the mean and standard deviation just presented may be used in conjunction with the envelope function to make an estimate of the full distribution of peak ground acceleration values. This simple approach to predicting the peak ground acceleration compares surprisingly well, given the simplicity of the model, with the purpose-built NGA models as may be appreciated from inspection of Figure 10. 

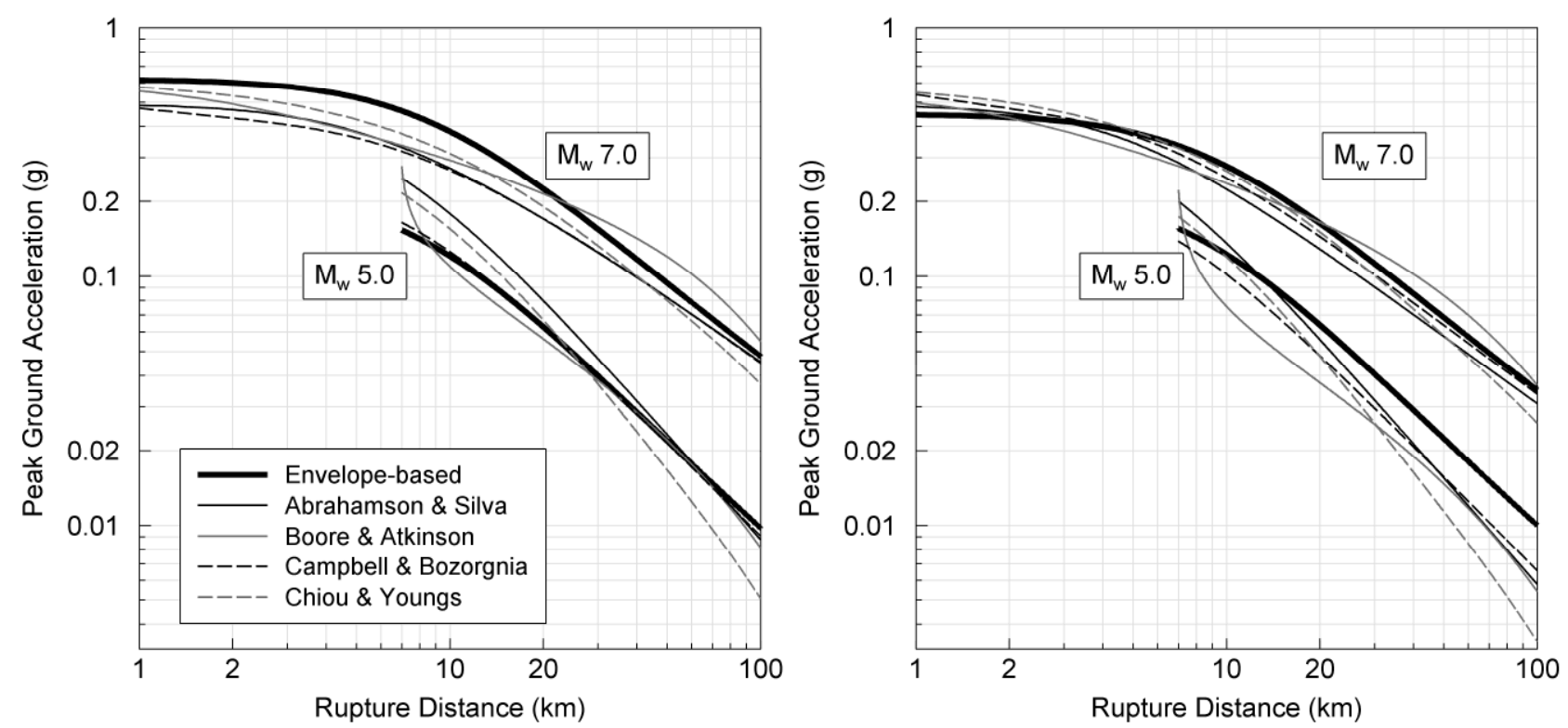

Figure 10: Comparison of the predictions of PGA using the approach outlined in this study and four of the NGA empirical ground-motion models: Abrahamson and Silva (2008), Boore and Atkinson (2008), Campbell and Bozorgnia (2008), and Chiou and Youngs (2008). The predictions shown here are for a site located perpendicular to a strike-slip fault. The $\mathrm{M}_{\mathrm{w}} 7.0$ event is surface rupturing while the depth to the top of the $M_{\mathrm{w}} 5.0$ event is $7 \mathrm{~km}$. The panel on the left depicts predictions for a site with a VS, $30=300 \mathrm{~m} / \mathrm{s}$ and depths to the $1 \mathrm{~km} / \mathrm{s}$ and $2.5 \mathrm{~km} / \mathrm{s}$ velocity horizons of $426 \mathrm{~m}$ and $2.05 \mathrm{~km}$ respectively. The panel on the right depicts the predictions for a site with VS,30 $=760 \mathrm{~m} / \mathrm{s}$ and depths to the $1 \mathrm{~km} / \mathrm{s}$ and $2.5 \mathrm{~km} / \mathrm{s}$ velocity horizons of $34 \mathrm{~m}$ and $640 \mathrm{~m}$ respectively.

The predictions of the various models shown in Figure 10 are generally very consistent. The most significant departures correspond to short distances for large events and the lower of the two considered average shear-wave velocities and at large distances for smaller events for the larger shear-wave velocity. In both cases, the departures are conservative and are also comparable with the general epistemic uncertainty associated with the NGA predictions themselves. Although Figure 10 only compares the median values of peak ground acceleration, the fact that the peak factor is weakly correlated with the shape parameters of the envelope function (correlations of between 0.20.3 are found) means that it is relatively straightforward to estimate the standard deviation associated with the logarithm of peak ground acceleration. We have seen that the peak factor is well modelled by a lognormal distribution and that ordinates of the envelope function itself are also lognormally distributed. Hence the product of the peak factor with the maximum value of the envelope function provides an estimate of the peak ground acceleration that should also be lognormally distributed. In order to estimate the logarithmic standard deviation of the peak ground acceleration estimates one may simply take the square root of the sum of the variance of the envelope function (evaluated at the time at which the function is a maximum) using Equation (18) or $(19)$ and the variance of the peak factor $\left(0.0439=0.2095^{2}\right)$. Because the model used herein to 
predict the Arias intensity has a logarithmic standard deviation that is a function of the site class, the magnitude of the event and the strength of the Arias intensity itself, no single value for the logarithmic standard deviation of the peak ground acceleration may be provided. However, when one calculates this value for magnitudes ranging from 5-8, for distances up to $100 \mathrm{~km}$ and for all site classes, one finds that the standard deviation of the logarithmic peak ground acceleration varies over the range [0.5,0.7] and that the variation of the actual values with respect to the various parameters (magnitude and ground-motion intensity) are strikingly similar to the NGA models.

\section{Summary and conclusions}

This work has addressed the problem of characterizing the time-varying amplitude of strong ground-motion. The primary purpose of computing such an envelope is to enable physically realistic stochastic accelerograms to be generated. However, the present study is only concerned with the prediction of an envelope of ground-motion amplitudes and in order to generate realistic stochastic accelerograms a robust model for the time-varying frequency content must also be available. The derivation of such a model is the topic of ongoing research.

Unlike many of the envelopes that have previously been proposed, the envelope function presented herein has been developed with seismic design consideration firmly in mind. We have presented alternative procedures that may be implemented when different analyses are required, i.e., either deterministic scenario-based analyses or analyses following on from PSHA. We have clearly demonstrated that the total uncertainty associated with the prediction of the envelope is greatly reduced when some knowledge of the spectral acceleration associated with a particular scenario is known.

The envelope function itself is energy-based in the sense that the overall intensity of the envelope is tied directly to the expected Arias intensity corresponding to the scenario for which we are generating the envelope. Furthermore, the shape of the envelope is based directly upon the Husid plot that depicts the temporal distribution of energy within an accelerogram. Although the Arias intensity is renowned to be a relatively unpredictable parameter, the formulation of the model allows the total uncertainty of the envelope amplitudes to be reduced to the extent that the model has relatively good predictive power. 
The envelope function is not generally able to predict the peak acceleration values of a signal. However, we have demonstrated that the relationship between the peak ground acceleration and the maximum amplitude of the envelope function follows a very well constrained lognormal distribution. Therefore, if one wishes to couple the envelope function presented herein with some estimate of the maximum departure from the envelope one is readily able to do so. The predictions of PGA that are obtained when this approach is adopted are very consistent with those of the recently developed NGA models.

\section{ACKNOWLEDGEMENTS}

Sara Sgobba wishes to express her gratitude to Prof. Mauro Mezzina for his cultural and economical support to the development of the present work. Peter Stafford is a fellow of the Willis Research Network and would like to acknowledge the generous financial support that is provided to him. 


\section{REFERENCES}

Abrahamson, N.A., and Silva, W.J., Summary of the Abrahamson \& Silva NGA groundmotion relations. Earthquake Spectra 2008. 24(1): p. 67-97.

Baker, J.W., and Cornell, C.A., Which spectral acceleration are you using? Earthquake Spectra 2006a. 22(2): p. 293-312.

Baker, J.W., and Cornell, C.A., Spectral shape, epsilon and record selection. Earthquake Engineering and Structural Dynamics 2006b. 35: p. 1077-1095.

Baker, J.W., Correlation of ground motion intensity parameters used for predicting structural and geotechnical response. Proceedings of the $10^{\text {th }}$ International Conference on Applications of Statistics and Probability in Civil Engineering (ICASP10), 2007, Tokyo, Japan.

Bazzurro, P., and Cornell, C.A., Disaggregation of Seismic Hazard. Bulletin of the Seismological Society of America 1999. 89(2): p. 501-520.

Benjamin, J.R., and Cornell, C.A., Probability, Statistics and Decision for Civil Engineers. McGraw-Hill, New York, 1970. 684p.

Beyer, K., and Bommer, J.J., Selection and scaling of real accelerograms for bi-directional loading: a review of current practice and code provisions. Journal of Earthquake Engineering 2007. 11(S1): p. 13-45.

Bommer, J.J., Stafford, P.J., and Alarcón, J.E., Empirical equations for the prediction of the Signifcant, Bracketed and Uniform duration of earthquake ground motion. Bulletin of the Seismoloical Society of America 2008. Under review.

Boore, D.M., and Atkinson, G.M., Ground-motion prediction equations for the average horizontal component of PGA, PGV, and 5\%-damped PSA at spectral periods between $0.01 \mathrm{~s}$ and 10.0 s. Earthquake Spectra 2008. 24(1): p. 99-138.

Campbell, K.W., and Bozorgnia, Y., NGA ground motion model for the geometric mean horizontal component of PGA, PGV, PGD and 5\% damped linear elastic response spectra for periods ranging from 0.01 to 10 s. Earthquake Spectra 2008. 24(1): p. 139-171.

Chiou, B.S.-J., Darragh, R., Gregor, N., and Silva, W., NGA project strong-motion database. Earthquake Spectra 2008. 24(1): p. 23-44.

Chiou, B.S.-J., and Youngs, R.R., An NGA model for the average horizontal component of peak ground motion and response spectra. Earthquake Spectra 2008. 24(1): p. 173-215.

Chouet, B., Aki, K., and Tsujiura, M., Regional variation of the scaling law of earthquake source spectra. Bulletin of the Seismological Society of America 1978. 68(1): p. 49-79.

Cramer, H., and Leadbetter, M.R., Stationary and related stochastic processes. Wiley, New York, 1967. 
Crandall, S.H., and Mark, W.D., Random vibration in mechanical systems. Academic Press, New York, 1963.

Di Paola, M., Transient spectral moments of linear systems. SM Archives 1985. 10: p. 225243.

Dobry, R., Idriss, I.M., and Ng, E., Duration characteristics of horizontal components of strong-motion earthquake records. Bulletin of the Seismological Society of America 1978. 68(5): p. 1487-1520.

Dugundji, J., Envelopes and pre-envelopes of real waveforms. IRE Transactions on Information Theory 1958. 4: p. 53-57.

Jangid, R.S., Response of SDOF system to non-stationary earthquake excitation. Earthquake Engineering and Structural Dynamics 2004. 33: p. 1417-1428.

Kanai, K., Some empirical formulas for the seismic characteristics of the ground. Bulletin of the Earthquake Research Institute, University of Tokyo 1957. 35: p. 309-325.

Langley, R.S., On various definitions of the envelope of a random process. Journal of Sound and Vibration 1986. 105(3): p.503-512.

Liang, J., Ray Chaudhuri, S., and Shinozuka, M., Simulation of nonstationary stochastic processes by spectral representation. Journal of Engineering Mechanics 2007. 133(6): p. 616-627.

Marple, S.L., Computing the discrete-time “analytic" signal via FFT. IEEE Transactions on Signal Processing 1999. 47(9): p.2600-2603.

Naeim, F., and Lew, M., On the use of design spectrum compatible time histories. Earthquake Spectra 1995. 11(1): p. 111-127.

Pinheiro, J., Bates, D., DebRoy, S., Sarkar, D., and the R Core team. nlme: linear and nonlinear mixed effects models. 2008. R package version 3.1-89.

Quek, S.T., Teo, Y.P., and Balendra, T., Non-stationary structural response with evolutionary spectra using seismological input model. Earthquake Engineering and Structural Dynamics 1990. 19: p. 275-288.

R Development Core Team. R: A language and environment for statistical computing. 2008. $\mathrm{R}$ Foundation for Statistical Computing, Vienna, Austria. ISBN 3-900051-07-0, URL http://www.R-project.org

Rezaeian, S., and Der Kiureghian, A., A stochastic ground motion model with separable temporal and spectral nonstationarities. Earthquake Engineering and Structural Dynamics 2008. 37: p. 1565-1584.

Soong, T.T., and Mircea, G., Random vibration of mechanical and structural systems. Prentice Hall, Englewood Cliffs, New Jersey, 1993. 415 p. 
Stafford, P.J., Berrill, J.B., and Pettinga, J.R., New predictive equations for Arias intensity from crustal earthquakes in New Zealand. Journal of Seismology 2008. doi 10.1007/s10950-0089114-2. available online.

Stafford, P.J., Alarcón, J.E., and Bommer, J.J., Empirical equations for the prediction of the equivalent number of cycles of earthquake ground motion. Soil Dynamics and Earthquake Engineering 2008a, under review.

Tajimi, H., A statistical method of determining the maximum response of a building structure during an earthquake. Proceedings of the Second World Conference on Earthquake Engineering, Tokyo and Kyoto 1960. Vol. II: p. 781-796.

Travasarou, T., Bray, J.D., and Abrahamson, N.A., Empirical attenuation relationship for Arias Intensity. Earthquake Engineering and Structural Dynamics 2003. 32: 1133-1155.

Zerva, A., Seismic source mechanisms and ground motion models. Probabilistic Engineering Mechanics 1988. 3(2): p. 64-74. 